\title{
Monosodium L-Glutamate and Dietary Fat Differently Modify the Composition of the Intestinal Microbiota in Growing Pigs
}

\author{
Ze-Meng Feng ${ }^{a}$ Tie-Jun $\mathrm{Li}^{\mathrm{a}} \mathrm{Li} \mathrm{Wu}^{\mathrm{a}}$ Ding-Fu Xiao ${ }^{\mathrm{b}}$ Francois Blachier ${ }^{\mathrm{c}}$ \\ Yu-Long Yin ${ }^{\mathrm{a}}$ \\ ${ }^{a}$ Institute of Subtropical Agriculture, The Chinese Academy of Sciences, Research Center \\ of Healthy Breeding Livestock \& Poultry, Hunan Engineering \& Research Center of Animal \\ \& Poultry Science, Key Lab Agro-Ecology Processing Subtropical Region, Scientific \\ Observational and Experimental Station of Animal Nutrition and Feed Science in South- \\ Central, Ministry of Agriculture, Changsha, Hunan, China; ${ }^{b}$ College of Animal Science and \\ Technology, Hunan Agricultural University, Changsha, Hunan, China; ' UMR914 INRA/ \\ AgroParisTech, Nutrition Physiology and Ingestive Behavior, Paris, France
}

\section{Key Words}

High-fat diet · Monosodium L-glutamate $\cdot$ Intestinal microbiota · Energy · Growing pig

\begin{abstract}
Background: The Chinese have been undergone rapid transition to a high-fat diet-consuming lifestyle, while monosodium L-glutamate (MSG) is widely used as a daily food additive. It has been reported that fat alters the composition of intestinal microbiota. However, little information is available on the effects of oral MSG on intestinal microbiota, and no study was done focusing on the interaction effect of fat and MSG with respect to intestinal microbiota. The present study thus aimed to determine the effects of MSG and/or fat on intestinal microbiota, and also to identify possible interactions between these two nutrients. Methods: Four isonitrogenous and iso-caloric diets were provided to growing pigs. The microbiota from jejunum, ileum, cecum, and colon were analyzed. Results: Our results show that both MSG and fat clearly increased the intestinal microbiota diversity. MSG and fat modified the composition of intestinal microbiota, particularly in the colon. Both MSG and fat promoted the colonization of microbes related to energy extraction in gastrointestinal tract via different ways. MSG promoted the colonization of Faecalibacterium prausnitzii and Roseburia, while fat increased the percentage of Prevotella in colon and other intestinal segments. Conclusion: Our results will help to understand how individual or combined dietary changes modify the microbiota composition to prevent obesity.


Feng et al.: Monosodium L-Glutamate and Dietary Fat Differently Modify the

Composition of the Intestinal Microbiota in Growing Pigs

\section{Introduction}

The mammals had co-evolved with microbes present in their gastrointestinal tracts in the past 160 million years. The intestinal microbiota represents at least about $10^{14}$ bacteria and approximately 500-1,000 species. The whole genome of intestinal microbiota is about 100 times bigger than the mammalian one [1]. The colonization of the intestine by these microbes is presumed to have consequences for the physiology and metabolism in mammals. Previous studies reported that intestinal microbiota influenced mammalian physiology and metabolism by acting on biological processes including angiogenesis, intestinal epithelial barrier function, and innate- and adaptive immunity [2-4]. Recent experimental evidence also pointed out the possible implication of changes in the intestinal microbiota composition as a possible determinant in some pathological processes including obesity, although the relative contribution of intestinal microbiota and other parameters, including calorieingestion and physical activity, in the etiology of obesity remains unknown.

Some countries, including China, have been characterized by an amazing development of industry and agronomical activities in recent years. And several metabolic diseases with previously low incidence have rapidly increased in these countries since then. Obesity is reaching pandemic proportions, especially in industrialized countries [5, 6]. According to the survey of the World Health Organization, there are about 700 million obese people and 2 billion overweight individuals around the world [7], which threaten people's health and bring great social burden notably in financial terms $[8,9]$. Various factors can induce obesity including environmental and genetic parameters $[10,11]$. For the reasons presented above, it is a challenge to control the spreading of obesity in China [12]. Excess dietary fat consumption is an important contributor of obesity related to high energy content, good palatability, and appetite stimulation [13]. The Chinese population, including children and adolescents, has undergone a rapid transition to a high-fat diet. Indeed, this change is occurring faster than the one observed in Western children over the last century $[14,15]$. Together with the high dietary fat consumption, in Asian countries, including China, monosodium L-glutamate (MSG) is traditionally added to food on a daily basis $[16,17]$. MSG consumption has been reported to be associated with obesity [18], but this finding remains highly controversial [19-22].

In recent studies, associations were found between the composition of intestinal microbiota and some metabolic diseases, e.g., obesity and type 2 diabetes [23, 24]. Overweight and obese individuals have a low diversity of intestinal microbiota $[25,26]$. Although the causal links between intestinal microbiota composition and obesity remain unclear, some working hypotheses are related to the effects of the intestinal microbiota on the nutrient digestion and absorption in the gastrointestinal tract [27], on the bile circulation between intestine and liver [28], on the triglyceride absorption, on the promotion of adipogenesis and reduction of lipolysis [29], on the induction of low-grade inflammation, and on interference with the intestinal barrier function [30,31]. By interfering with metabolism and intestinal microbiota immune regulatory networks, intestinal microbiota may have a positive or negative influence on obesity [32]. For instance, it had been reported that an increased amount of Lactobacillus [33], Staphylococcus aureus [34], Escherichia coli [35], and some other species may favor obesity.

The composition of intestinal microbiota is known to be affected by diverse factors, such as temperature, dietary contents, use of antibiotics etc. [36]. Although some studies have been done to evaluate the influence of dietary fat on the composition of intestinal microbiota [37, 38], little is known regarding the effect of MSG on intestinal microbiota, and, to our knowledge, there is no published data on the influence of MSG and dietary fat ingestion on the intestinal microbiota composition. Pigs are considered to be a suitable animal model for studying human nutrition due to their apparent similarities to humans; i.e., both species are omniv- 
Feng et al.: Monosodium L-Glutamate and Dietary Fat Differently Modify the

Composition of the Intestinal Microbiota in Growing Pigs

orous, and they share nutritional, digestive, hematological and cardiovascular characteristics [39]. In the present study, MSG was provided to growing pigs at a concentration that was not vastly different from that found in human food, with a consideration of the dietary fat content. The aim of this study was thus to determine the effect of an oral daily supplementation with MSG and/or fat on the composition of porcine intestinal microbiota through Denaturing Gradient Gel Electrophoresis (DGGE) and quantitative PCR quantification.

\section{Material and Methods}

Experimental Design, Animals, and Diets

A $2 \times 2$ factorial design was used in the present study. A total of 32 growing pigs (crossbred population composed of York, maternal Landrace, and Duroc breeds; average body weight $25 \pm 1.3 \mathrm{~kg}$ ) from 4 litters were used. The pigs were randomly divided into four groups (8 repeats), half male and half female per group. Four iso-nitrogenous and iso-caloric diets (basal diet (BD); high fat diet (HF); basal diet with 3\% MSG (BDM), and high-fat diet with 3\% MSG (HFM)) were provided to growing pigs. The BD group acts as control. The detailed compositions of the four diets are shown in supplemental table 1 (supplemental material available at http://content.karger.com/ProdukteDB/produkte.asp?doi=380889). The four diets and water were provided to the test pigs in ad libitum as MSG is related with food intake regulation. 30 days later, the pigs were weighed and then sacrificed by jugular puncture under general anesthesia via the intravenous injection of $4 \%$ sodium pentobarbital solution ( $40 \mathrm{mg} / \mathrm{kg}$ body weight). The average daily gain and carcass segmentation test was performed. Analysis on the composition of longissimus dorsi was also performed. The contents of jejunum, ileum, cecum, and colon from each pig were collected and then immediately stored at $-20^{\circ} \mathrm{C}$ until analysis. All experimental procedures used in this study were approved by the Animal Care and Use Committee of the Chinese Academy of Sciences.

\section{DNA Extraction}

The metagenomic DNAs of intestinal microbiota were isolated from $250 \mathrm{mg}$ of contents of jejunum, ileum, cecum, and colon using The QIAmp DNA Stool Mini kit (Qiagen, Hilden, Germany) following the protocol of 'Isolation of DNA from Larger Volumes of Stool' described in QIAamp ${ }^{\circledR}$ DNA Stool Handbook (2nd ed.). Purified DNA samples were eluted in a final volume of $50 \mu$ with $\mathrm{ddH}_{2} \mathrm{O}$, and the qualities and concentrations of purified DNAs were determined by spectrophotometry using NanoDrop ${ }^{\circledR}$ ND2000 (NanoDrop Technologies Inc., Wilmington, DE, USA). Purified DNAs were stored at $-70{ }^{\circ} \mathrm{C}$ until analysis.

\section{DGGE Profiles of Intestinal Microbiota}

The purified microbial genomic DNA was amplified by polymerase chain reaction (PCR) targeting the V3 region of the 16S rDNA gene and using the universal bacterial primers HDA1-GC-F and HAD-2-R [40] (HDA1-GC-F, 5'-CGCCCGGGGCGCGCCCCGGGCGGGGCGGGGGCACGGGGGGACTCCTACGGGAGGCAGCAG-3' and HAD-2-R, 5'-GTATTACCGCGGCTGCTGGCA-3'). The PCR mixture (50 $\mu \mathrm{l}$ total volume) contained $25 \mu \mathrm{l}$ of $2 \times$ Fermentas PCR master mix (Thermo Fisher Scientific, Waltham, MS, USA), $22 \mu \mathrm{l}$ of $\mathrm{ddH}_{2} \mathrm{O}, 1 \mu \mathrm{l}$ microbial genomic DNA and $1 \mu \mathrm{l}(10 \mathrm{nmol} / \mathrm{l})$ of each primers. The PCR reaction was performed by using Eppendorf master cycler (Eppendorf, Hamburg, Germany) with the following parameters: initial denaturation at $94{ }^{\circ} \mathrm{C}$ for $5 \mathrm{~min} ; 30$ cycles of denaturation at $94{ }^{\circ} \mathrm{C}$ for $30 \mathrm{~s}$, annealing at $56^{\circ} \mathrm{C}$ for $30 \mathrm{~s}$, elongation at $72{ }^{\circ} \mathrm{C}$ for 2 $\mathrm{min}$, and final elongation at $72{ }^{\circ} \mathrm{C}$ for $10 \mathrm{~min}$; then the probes were cooled at $4{ }^{\circ} \mathrm{C}$. The PCR products were identified by electrophoresis at $120 \mathrm{~V}$ for $50 \mathrm{~min}$ on a $2 \%(\mathrm{~m} / \mathrm{v})$ agarose gel, and then stored at $-20^{\circ} \mathrm{C}$ until DGGE analysis.

Electrophoresis was performed with a D-Code universal mutation detection system (Bio-Rad, Hercules, CA, USA) using $8 \%(\mathrm{~m} / \mathrm{v}$ ) polyacrylamide gels (37.5:1, acrylamide: bisacrylamide) with a 35-65\% gradient of $7 \mathrm{~mol} / \mathrm{l}$ urea and $40 \% \mathrm{v} / \mathrm{v}$ ) formamide. Electrophoretic runs were done in a Tris-acetate-EDTA buffer (40 $\mathrm{mmol} / \mathrm{l}$ Tris, $20 \mathrm{mmol} / \mathrm{l}$ acetic acid, and $1 \mathrm{mmol} / \mathrm{l}$ EDTA) at $200 \mathrm{~V}$ for $10 \mathrm{~min}$ and then $120 \mathrm{~V}$ for $16 \mathrm{~h}$. Gels were stained with ethidium bromide $(1 \%)$ and then viewed by a Molecular Imager ${ }^{\circledR}$ ChemiDocTM XRS+ instrument (Bio-Rad). DGGE profiles were compared by determining the migration and peak density value of bands using Quantity One 4.62 software (Bio-Rad), while the tolerance was set as 1\%.The similarity system tree diagram was built using unweighted pair-group method with arithmetic means (UPGMA) clustering. The diversity of intestinal microbiota was evaluated through three indexes: $\mathrm{i})$ the Shannon-Wiener index $\left(\mathrm{H}^{\prime}=\right.$ 
Feng et al.: Monosodium L-Glutamate and Dietary Fat Differently Modify the

Composition of the Intestinal Microbiota in Growing Pigs

$-\Sigma(\mathrm{ni} / \mathrm{N}) \ln (\mathrm{ni} / \mathrm{N}))$, ii) the Simpson dominance index $\left(\lambda=\Sigma(\mathrm{ni} / \mathrm{N})^{2}\right)$, iii) the evenness index $\left(\mathrm{e}=\mathrm{H}^{\prime} / \operatorname{lnS}\right)$, with $\mathrm{S}$ being the total bands number, ni being peak density value of bands, and $\mathrm{N}$ being the sum of peak density value of all bands.

Cloning and Sequencing of Bands in Polyacrylamide Gels after DGGE

All different bands visualized in polyacrylamide gels after DGGE were extracted and placed in TE buffer at $4{ }^{\circ} \mathrm{C}$ overnight. The extracted DNAs were amplified by RT-PCR, and the PCR products were processed for the DGGE analysis to identify the different bands. The amplification products were purified using a Promega Wizard SV Gel and PCR Clean-up System (Promega Corporation, Madison, WI, USA) and then be linked to pGEM-T Easy (Promega Corporation). The plasmids were introduced into competent DH5 $\alpha$ cells and incubated overnight at $37^{\circ} \mathrm{C}$. Cells that contained successfully constructed plasmids were obtained using an X-Gal screening test. The plasmids were obtained using EZ-10 Spin Column Plasmid DNA Minipreps Kit (BBI, Shanghai, China) according to the recommended protocol and then sequenced at BGI (Beijing, China). The partial-length $16 \mathrm{~S}$ rDNA gene sequences were directly compared with corresponding sequences in the $16 \mathrm{~s}$ ribosomal RNA sequences (Bacteria and Archaea) databank via National Center for Biotechnology Information using BLASTN.

Quantitative PCR Quantification of Intestinal Microbiota

Quantitative PCR was performed to further quantitatively evaluate the effect of dietary supplementation with fat and MSG on the subpopulation of species related to energy extraction using group-specific primers. The following 16 microbial genera were previously reported to be associated with energy harvesting: Bacteroidetes, Bacteroides fragilis, Bacteroides thetaiotaomicron, Firmicutes, Akkermansia muciniphila, Clostridium coccoides group, Clostridium leptum subgroup, Clostridium difficile, Clostridium clostridiiforme, Clostridium coccoides eubacteria, Faecalibacterium prausnitzii, Fusobacterium prausnitzii, Methanobrevibacter smithii, Peptostreptococcus productus, Prevotella, and Roseburia. Quantitative PCR was carried out using species- and group-specific primers of intestinal microbiota that target 16S rDNA genes (supplemental table 2 (supplemental material available at http://content.karger.com/ProdukteDB/produkte. $a s p$ ?doi=380889)). The quantitative results were normalized by contrasting to the total intestinal microbiota. The dilutions were added to a final volume of $25 \mu$ l. Amplification and detection of DNA by RT-PCR was performed with ABI PRISM 7900 HT (Applied Biosystems, Foster City, CA, USA) using 384-well plates. Duplicate sample analysis was routinely performed in a total volume of $25 \mu$ l using SYBR ${ }^{\circledR}$ Premix Ex TaqTM II (TAKARA, Dalian, China). Data from duplicate samples were analyzed using the ABI 7900 SDS software (version 2.3; Applied Biosystems).

\section{Statistical Analyses}

Principal component analysis (PCA) was done based on peak density value of bands using PC-ORD program, and the results were obtained using the princomp program of SAS 9.2 (SAS Institute Inc., Cary, NC, USA). The data are expressed as means \pm SEM. Differences between groups were assessed by ANOVA. Statistical analyses were performed with SAS 9.2. The differences were considered as statistically significant for $\mathrm{p}<0.05$.

\section{Results}

MSG and Dietary Fat Promoted Fat Deposition in Muscle in Growing Pigs

MSG and dietary fat had no obvious effects on average daily gain and food intake of pigs. The parameters used to evaluate carcass segmentation, containing slaughter yield, back fat thickness, lion-eye area, total fat and total skeletal muscle, were also not effected by dietary supplementation of MSG and dietary fat (data not shown). The result of longissimus dorsi composition analysis was shown in figure 1. Both dietary supplementation of MSG and fat had no effect on crude protein and dry matter. Surprisingly, MSG and fat promote fat deposition in longissimus dorsi, and an obviously synergistic effect was found when the two nutrients were given in one diet. 
Feng et al.: Monosodium L-Glutamate and Dietary Fat Differently Modify the Composition of the Intestinal Microbiota in Growing Pigs

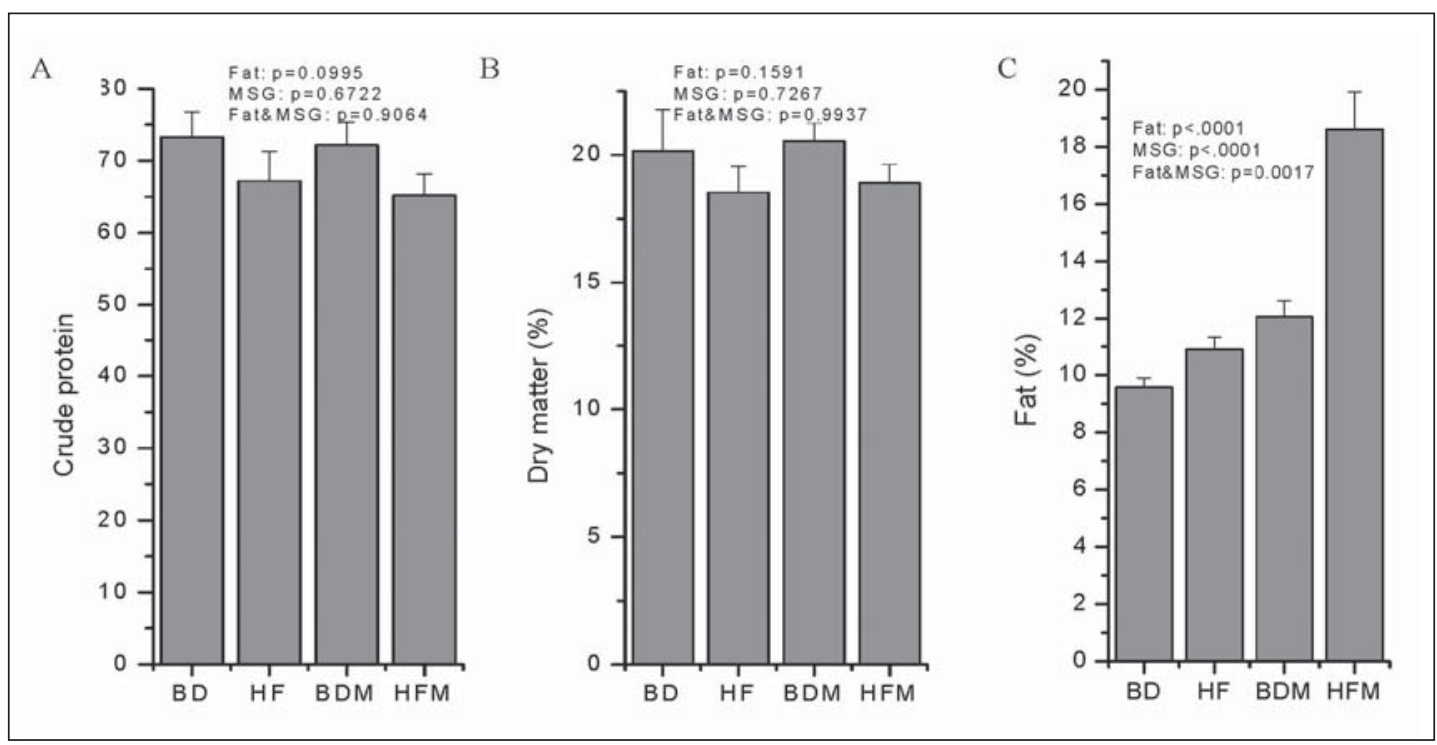

Fig. 1. Dietary supplementation of MSG and fat can influence the main components in muscle of growing pigs $(\mathrm{n}=4)$. A Crude protein, $\mathbf{B}$ dry matter, $\mathbf{C}$ fat.

Table 1. Diversity indexes of gut microbial community

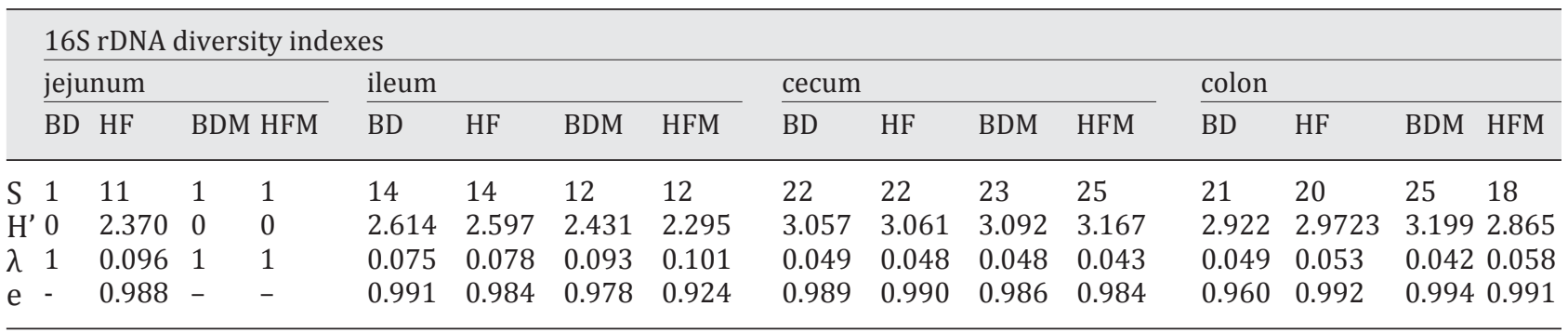

$\mathrm{S}=$ Bands number; $\mathrm{H}^{\prime}=$ Shannon-Wiener index; $\lambda=$ Simpson dominance index; $\mathrm{e}=$ evenness index; $\mathrm{BD}=\mathrm{basal}$ diet, $\mathrm{HF}=\mathrm{high}$ fat diet, $\mathrm{BDM}=$ basal diet + MSG, HFM = high fat diet + MSG.

MSG and Dietary Fat Change the Composition of the Intestinal Microbiota in Growing Pigs

In the present study, the influences of dietary fat and MSG on intestinal microbiota in growing pigs were determined through DGGE. The results are shown in figure 2 and table 1. Dietary fat increased microbial diversity in the jejunum, and MSG counteracted this change when added to dietary fat. As for ileum, both dietary fat and MSG decrease the diversity of ileal microbiota; the two dietary supplements have synergistic effects. On the other hand, both dietary fat and MSG increase the diversity of cecal microbiota; here too, the two dietary supplements have synergistic effects. Interestingly, both dietary fat and MSG increase the diversity of colonic microbiota when given individually, but the two nutrients have antagonistic effects when given together. In order to further evaluate the effect of dietary fat and MSG on intestinal microbiota, PCA was done based on band peak density value. The PCA results are shown in figure 3. MSG has a marked effect on ileal microbiota, while dietary fat and MSG have less effect on intestinal microbiota when given together. It is worth noting that with respect to the diversity of colonic microbiota some interactions between dietary fat and MSG are apparent. 
Feng et al.: Monosodium L-Glutamate and Dietary Fat Differently Modify the

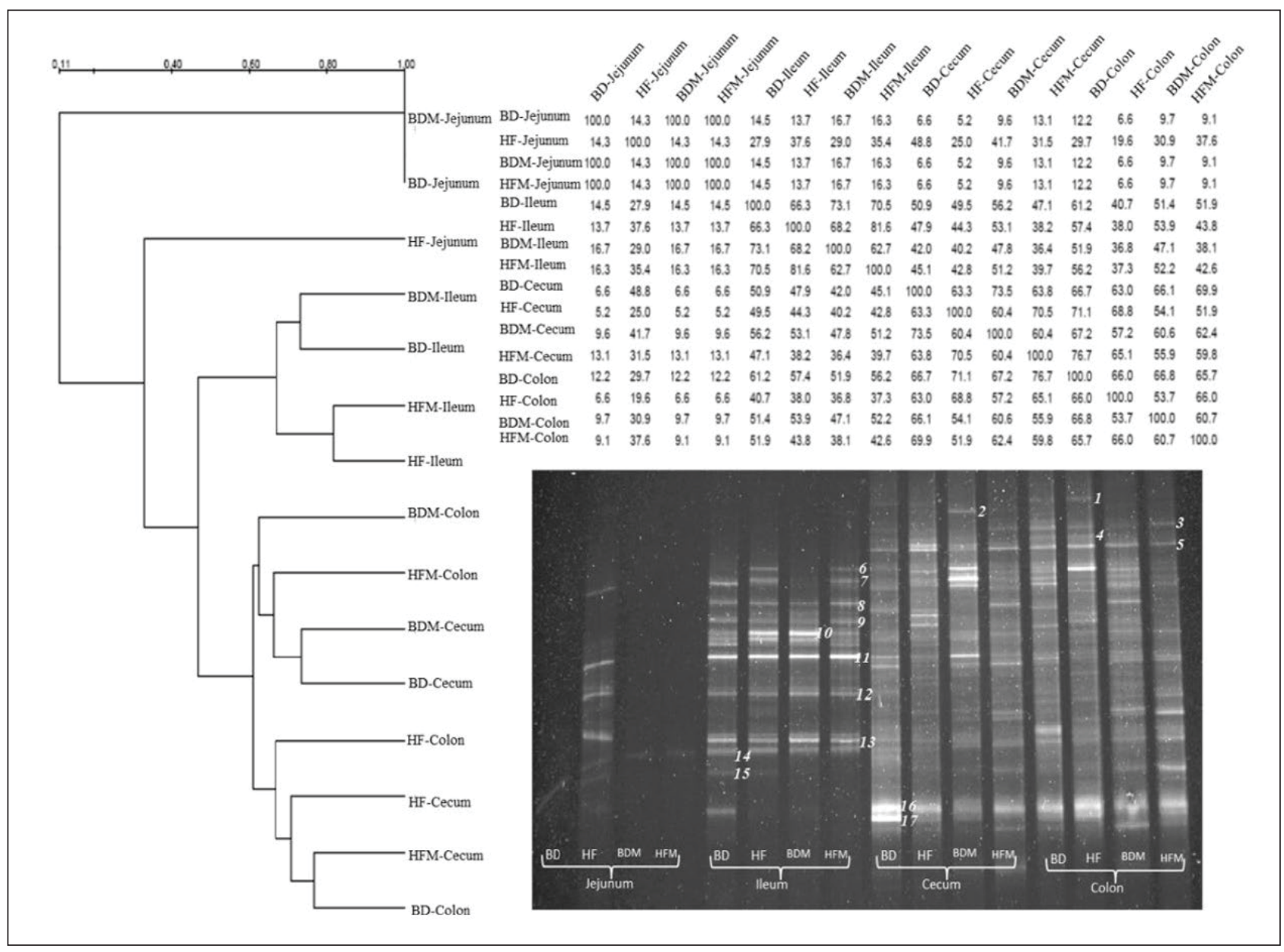

Fig. 2. PCR-DGGE profiles of intestinal contents using the following primer sets of intestinal microbiota: HAD1 GC and HAD-2 (35-65\% DGGE). The clustering dendrogram was generated with Quantity One software using the UPGMA method. 1) Holdemaniafiliformis, 2) Escherichia fergusonii, 3) Ruminococcuscallidus, 4) Clostridium hylemonae, 5) Allisonellahistaminiformans, 6) Actinobacillusarthritidis, 7) Actinobacillus minor, 8) Clostridium chauvoei, 9) Clostridium chauvoei, 10) Clostridium chauvoei, 11) Clostridium glycolicum, 12) Shigellaflexneri, 13) Salmonella enterica subsp., 14) Roseburiafaecis, 15) Pseudomonas geniculate, 16) Enterobacter cloacae, 17) Lactobacillus taiwanensis.

Fig. 3. Corresponding PCA analysis of intestinal microbiota. 1) BDileum, 2) HF-ileum, 3) BDM-ileum, 4) HFM-ileum, 5) BD-cecum, 6) HF-cecum, 7) BDM-cecum, 8) HFM-cecum, 9) BD-colon, 10) HFcolon, 11) BDM-colon, 12) HFMcolon.

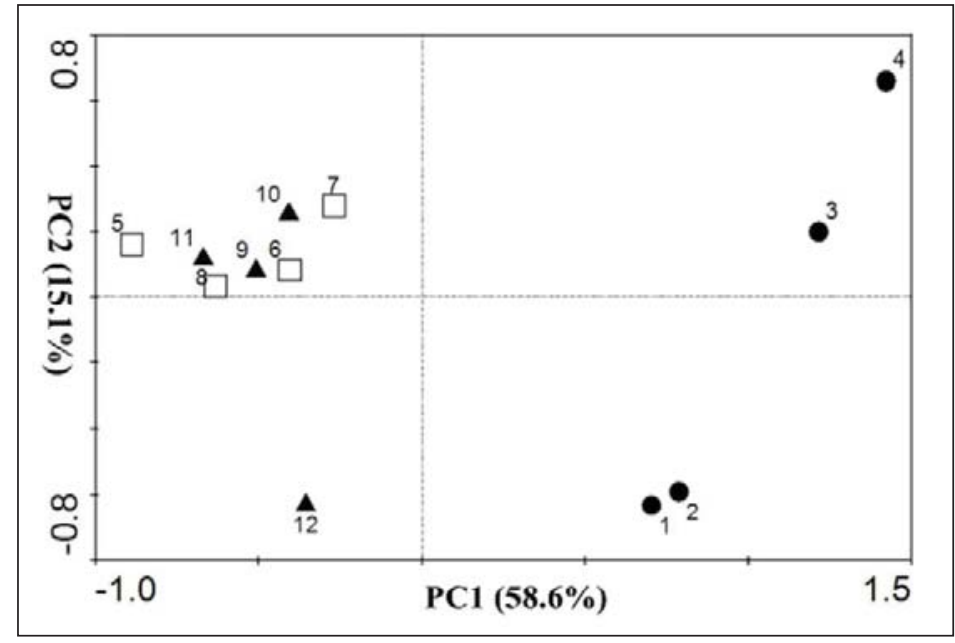


Feng et al.: Monosodium L-Glutamate and Dietary Fat Differently Modify the

Composition of the Intestinal Microbiota in Growing Pigs

Table 2. Changes of preponderant and microbial genus related to energy extraction in jejunum induced by supplementation with fat and/or MSG $(n=4)$

\begin{tabular}{|c|c|c|c|c|c|c|c|}
\hline \multirow[t]{2}{*}{ Species } & \multicolumn{4}{|c|}{ Abundants/all bacteria $\%$ (mean $\pm \mathrm{SD})$} & \multicolumn{3}{|c|}{ Analysis of variance ( $\mathrm{p}$ values) } \\
\hline & $\mathrm{BD}$ & $\mathrm{HF}$ & BDM & HFM & fat effect & GSM effect & interaction \\
\hline Firmicutes & $3.5120 \pm 0.9797$ & $6.3907 \pm 2.4155$ & $2.842756 \pm 0.4754$ & $5.3374 \pm 1.6080$ & 0.1330 & 0.6218 & 0.9145 \\
\hline Bacteroidetes & $20.6380 \pm 6.3896$ & $22.6909 \pm 8.7698$ & $17.4051 \pm 7.8590$ & $37.8840 \pm 9.8415$ & 0.1848 & 0.5353 & 0.2743 \\
\hline Peptostreptococcus productus & $0.9282 \pm 0.1057$ & $1.1448 \pm 0.4551$ & $0.5515 \pm 0.1275$ & $0.3832 \pm 0.1674$ & 0.7130 & 0.0432 & 0.4685 \\
\hline Clostridium coccoides group & $0.8033 \pm 0.5710$ & $0.2970 \pm 0.0735$ & ND & $0.7533 \pm 0.3846$ & - & - & - \\
\hline Prevotella & $0.0125 \pm 0.0013$ & $0.4394 \pm 0.0668$ & ND & $0.3648 \pm 0.0954$ & - & - & - \\
\hline
\end{tabular}

$\mathrm{BD}=$ Basal diet; $\mathrm{HF}=$ high fat diet; $\mathrm{BDM}=$ basal diet $+\mathrm{MSG}, \mathrm{HFM}=$ high fat diet + MSG; ND = not detected.

Table 3. Changes of preponderant and microbial genus related to energy extraction in ileum induced by supplementation with fat and/or MSG $(\mathrm{n}=4)$

\begin{tabular}{|c|c|c|c|c|c|c|c|}
\hline \multirow[t]{2}{*}{ Species } & \multicolumn{4}{|c|}{ Abundants/all bacteria $\%$ (mean $\pm \mathrm{SD})$} & \multicolumn{3}{|c|}{ Analysis of variance ( $p$ values) } \\
\hline & BD & $\mathrm{HF}$ & BDM & HFM & fat effect & GSM effect & interaction \\
\hline Firmicutes & $1.9035 \pm 0.2189$ & $11.1943 \pm 1.4881$ & $4.3741 \pm 1.1205$ & $2.2283 \pm 0.7357$ & 0.0501 & 0.0239 & $<0001$ \\
\hline Bacteroidetes & $23.0815 \pm 3.2919$ & $18.6503 \pm 8.2716$ & $35.2630 \pm 7.7761$ & $59.6959 \pm 11.9835$ & 0.3887 & 0.0085 & 0.0839 \\
\hline Peptostreptococcus productus & $0.5686 \pm 0.1941$ & $1.6744 \pm 0.5641$ & $0.3647 \pm 0.2018$ & $3.1662 \pm 1.9060$ & 0.0300 & 0.6491 & 0.4935 \\
\hline Prevotella & ND & $0.0443 \pm 0.0124$ & $1.9669 \pm 0.3512$ & ND & - & - & - \\
\hline
\end{tabular}

$\mathrm{BD}=$ Basal diet; $\mathrm{HF}=$ high fat diet; $\mathrm{BDM}=$ basal diet $+\mathrm{MSG}, \mathrm{HFM}=$ high fat diet + MSG; ND = not detected.

\section{MSG and Dietary Fat Markedly Change the Percentages of Energy Harvesting-Related}

Species in Total Intestinal Microbiota in Growing Pigs

Using DGGE technology, the general changes of intestinal microbial composition can be determined, but some species that represent less than $1 \%$ of the total intestinal microbiota cannot be detected. After broadly literature review, the percentages of 17 energy harvestingrelated species within the total intestinal microbiota were further determined by RT-PCR using group-specific primers. The result of cloning and sequencing of bands after DGGE show that most of changes are related to energy harvesting-related species.

Less microbial species were detected in the jejunum. Indeed, only 6 out of the 17 species were detected in this intestinal part. The results are shown in table 2. Furthermore, our results indicate that addition of dietary fat decrease the percentage of the jejunal Clostridium coccoides group while increasing the percentages of jejunal Prevotella. Dietary supplementation with MSG markedly decreased the percentages of jejunal Peptostreptococcus productus ( $\mathrm{p}=0.0432$ ), Prevotella, and Clostridium coccoides group.

Similarly, there were In the ileum also only few microbial species were present. As indicated in table 3 , only 4 out of the 17 species were detected. Our results indicate that addition of dietary fat increased the percentages of ileal Peptostreptococcus productus $(\mathrm{p}=0.0300)$ and Prevotella. Dietary supplementation with MSG markedly increased the percentages of ileal Firmicutes $(\mathrm{p}=0.0239)$, Bacteroidetes $(\mathrm{p}=0.0085)$, and Prevotella. When dietary supplementation with both fat and MSG was done, the two nutrients have antagonistic effects on Firmicutes $(\mathrm{p}<0.0001)$.

As expected, an increased number of microbial species were found in the cecum when compared with the data obtained in the small intestine. Little impact on the percentages of species with energy extraction capacity was found when giving dietary fat and MSG together (table 4). Addition of dietary fat increase the percentages of cecal Prevotella $(\mathrm{p}=0.0490)$ and 
Feng et al.: Monosodium L-Glutamate and Dietary Fat Differently Modify the

Composition of the Intestinal Microbiota in Growing Pigs

Table 4. Changes of preponderant and microbial genus related to energy extraction in cecum induced by supplementation with fat and/or MSG $(\mathrm{n}=4)$

\begin{tabular}{|c|c|c|c|c|c|c|c|}
\hline \multirow[t]{2}{*}{ Species } & \multicolumn{4}{|c|}{ Abundants/all bacteria $\%$ (mean \pm SD) } & \multicolumn{3}{|c|}{ Analysis of variance ( $p$ values) } \\
\hline & $\mathrm{BD}$ & $\mathrm{HF}$ & BDM & HFM & fat effect & GSM effect & interaction \\
\hline Bacteroidetes & $69.4465 \pm 8.7494$ & $68.8480 \pm 4.0937$ & $82.9468 \pm 5.7753$ & $58.6386 \pm 11.0435$ & 0.1103 & 0.7045 & 0.1303 \\
\hline Firmicutes & $33.2687 \pm 6.0711$ & $31.5021 \pm 4.5565$ & $24.3763 \pm 3.1377$ & $26.8105 \pm 6.5385$ & 0.9508 & 0.2269 & 0.6984 \\
\hline Bacteroides fragilis & $0.0031 \pm 0.0018$ & $0.0572 \pm 0.0164$ & $0.0038 \pm 0.0009$ & $0.0032 \pm 0.0015$ & 0.1237 & 0.1518 & 0.3118 \\
\hline Bacteroides thetaiotaomicron & $0.0039 \pm 0.0029$ & $0.0068 \pm 0.0025$ & $0.0251 \pm 0.0230$ & $0.0018 \pm 0.0010$ & 0.2381 & 0.5557 & 0.1560 \\
\hline Clostridium coccoides group & $3.1156 \pm 1.7242$ & $4.6772 \pm 1.1390$ & $2.5195 \pm 0.9287$ & $5.4637 \pm 0.9423$ & 0.0932 & 0.9408 & 0.5905 \\
\hline Clostridium leptum subgroup & $3.0298 \pm 1.1952$ & $1.4676 \pm 0.6432$ & $2.2224 \pm 0.8187$ & $2.2066 \pm 0.4821$ & 0.3298 & 0.9397 & 0.3878 \\
\hline Fusobacterium prausnitzii & $0.0596 \pm 0.0198$ & $0.0706 \pm 0.0239$ & $0.1122 \pm 0.0524$ & $0.2101 \pm 0.0984$ & 0.4188 & 0.1204 & 0.4901 \\
\hline Methanobrevibacter smithii & $0.0083 \pm 0.0050$ & $0.0041 \pm 0.0011$ & $0.0015 \pm 0.0005$ & $0.0017 \pm 0.0004$ & 0.5912 & 0.0507 & 0.3401 \\
\hline Peptostreptococcus productus & $0.8705 \pm 0.5207$ & $0.1096 \pm 0.0572$ & $0.8530 \pm 0.3845$ & $0.7547 \pm 0.3455$ & 0.2370 & 0.3517 & 0.3592 \\
\hline Faecalibacterium prausnitzii & $1.4241 \pm 0.7306$ & $0.6435 \pm 0.2047$ & $1.4751 \pm 0.5340$ & $2.3984 \pm 0.9853$ & 0.9168 & 0.1928 & 0.2185 \\
\hline Prevotella & $0.1403 \pm 0.0844$ & $2.7196 \pm 1.2531$ & $0.5815 \pm 0.2330$ & $6.3986 \pm 3.1962$ & 0.0490 & 0.2582 & 0.4313 \\
\hline Roseburia & $0.0233 \pm 0.0164$ & $0.1148 \pm 0.0358$ & $0.1140 \pm 0.0507$ & $0.4885 \pm 0.1354$ & 0.0223 & 0.0037 & 0.0851 \\
\hline
\end{tabular}

$\mathrm{BD}=$ Basal diet; $\mathrm{HF}$ = high fat diet; $\mathrm{BDM}=$ basal diet + MSG, HFM = high fat diet + MSG; ND = not detected.

Table 5. Changes of preponderant and microbial genus related to energy extraction in colon induced by supplementation with fat and/or MSG $(n=4)$

\begin{tabular}{|c|c|c|c|c|c|c|c|}
\hline \multirow[t]{2}{*}{ Species } & \multicolumn{4}{|c|}{ Abundants/all bacteria $\%$ (mean $\pm \mathrm{SD})$} & \multicolumn{3}{|c|}{ Analysis of variance ( $p$ values) } \\
\hline & $\mathrm{BD}$ & $\mathrm{HF}$ & BDM & HFM & fat effect & GSM effect & interaction \\
\hline Firmicutes & $26.2944 \pm 3.6063$ & $22.9064 \pm 2.3034$ & $30.0668 \pm 2.9462$ & $48.3318 \pm 4.6283$ & 0.0298 & 0.0005 & 0.0061 \\
\hline Bacteroidetes & $37.0751 \pm 5.5998$ & $45.9538 \pm 7.1753$ & $43.0762 \pm 6.7602$ & $59.2038 \pm 11.7137$ & 0.1345 & 0.2342 & 0.6568 \\
\hline Bacteroides fragilis & $0.0051 \pm 0.0022$ & $0.0144 \pm 0.0076$ & $0.0048 \pm 0.0013$ & $0.0068 \pm 0.0062$ & 0.3818 & 0.5429 & 0.7147 \\
\hline Bacteroides thetaiotaomicron & $0.0094 \pm 0.0048$ & $0.0035 \pm 0.0028$ & $0.0084 \pm 0.0081$ & ND & - & - & - \\
\hline Clostridium coccoides group & $5.1648 \pm 1.2656$ & $11.2793 \pm 2.2489$ & $5.8250 \pm 1.1229$ & $6.0493 \pm 1.1818$ & 0.0471 & 0.1456 & 0.0638 \\
\hline Clostridium leptum subgroup & $0.6690 \pm 0.0777$ & $1.6621 \pm 0.2370$ & $1.7190 \pm 0.2297$ & $0.9482 \pm 0.1276$ & 0.4143 & 0.5816 & 0.0002 \\
\hline Prevotella & $3.9999 \pm 1.2033$ & $2.9679 \pm 0.6942$ & $2.9383 \pm 0.3578$ & $4.7990 \pm 0.7318$ & 0.8601 & 0.6197 & 0.1302 \\
\hline Faecalibacterium prausnitzii & $0.9160 \pm 0.0935$ & $0.9601 \pm 0.1620$ & $2.8883 \pm 0.4459$ & $1.7118 \pm 0.3104$ & 0.0423 & 0.0001 & 0.0495 \\
\hline Fusobacterium prausnitzi & $0.1533 \pm 0.0401$ & $0.1929 \pm 0.0243$ & $0.8358 \pm 0.0772$ & $0.2938 \pm 0.0543$ & $<0.0001$ & $<0.0001$ & $<0.0001$ \\
\hline Peptostreptococcus productus & $5.0210 \pm 0.4358$ & $1.8800 \pm 0.6580$ & $1.4813 \pm 0.1410$ & $0.6200 \pm 0.1478$ & 0.0002 & $<.0001$ & 0.0149 \\
\hline Methanobrevibacter smithii & $0.0285 \pm 0.0141$ & $0.1144 \pm 0.0329$ & $0.0121 \pm 0.0035$ & $0.0120 \pm 0.0027$ & 0.0404 & 0.0033 & 0.0359 \\
\hline Roseburia & $1.9507 \pm 0.4530$ & $0.9430 \pm 0.2559$ & $2.0390 \pm 0.5520$ & $0.6523 \pm 0.1776$ & 0.0063 & 0.7478 & 0.6514 \\
\hline
\end{tabular}

$\mathrm{BD}=$ Basal diet; HF = high-fat diet; BDM = basal diet + MSG; HFM: high-fat diet + MSG; ND not detected.

Roseburia $(\mathrm{p}=0.0223)$. When dietary supplementation with both fat and MSG was given, no interaction of the two nutrients was observed.

In the colon, again as expected, the microbial species were much more abundant than in the small intestine. Dietary supplementation with fat and MSG together markedly influenced the percentages of species with energy extraction capacity (table 5). We observed that the addition of dietary fat decreased the percentages of colonic Firmicutes ( $\mathrm{p}=0.0298)$, Bacteroides thetaiotaomicron, Peptostreptococcus productus $(\mathrm{p}=0.0002)$, and Roseburia $(\mathrm{p}=$ $0.0063)$ while increasing the percentages of colonic Clostridium coccoides group $(\mathrm{p}=0.0471)$, Faecalibacterium prausnitzii $(\mathrm{p}=0.0423)$, Fusobacterium prausnitzii $(\mathrm{p}<0.0001)$, and Methanobrevibacter smithii $(\mathrm{p}=0.0404)$. Dietary supplementation with MSG markedly decreased the percentages of colonic Bacteroides thetaiotaomicron, Peptostreptococcus productus $(\mathrm{p}<$ $0.0001)$, and Methanobrevibacter smithii $(\mathrm{p}=0.0033)$ while increasing the percentages of colonic Firmicutes ( $p=0.0005)$, Faecalibacterium prausnitzii $(p=0.0001)$, and Fusobacterium prausnitzii $(\mathrm{p}<0.0001)$. When dietary supplementation was performed with both fat and 
Feng et al.: Monosodium L-Glutamate and Dietary Fat Differently Modify the

Composition of the Intestinal Microbiota in Growing Pigs

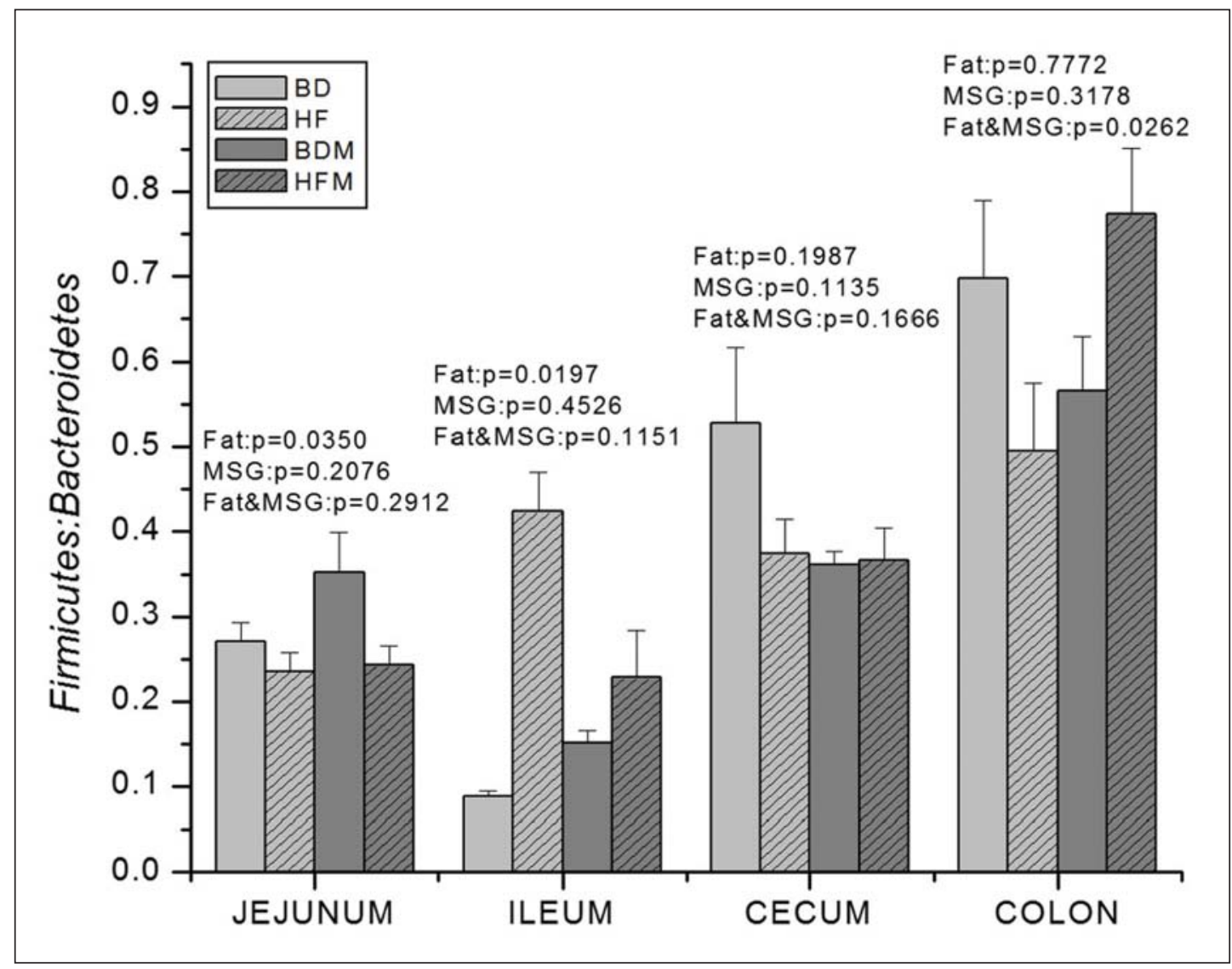

Fig. 4. Dietary MSG and fat influence the ratio of Firmicutes/Bacteroidetes in the gastrointestinal tract of growing pigs $(n=4)$.

MSG, the two nutrients synergistically decreased the percentage of colonic Peptostreptococcus productus ( $\mathrm{p}=0.0149$ ) while having antagonistic effect on the colonic Firmicutes $(\mathrm{p}=$ $0.0061)$, Clostridium leptum subgroup $(\mathrm{p}=0.0002)$, Faecalibacterium prausnitzii $(\mathrm{p}=0.0495)$, Fusobacterium prausnitzii ( $<<0.0001)$, and Methanobrevibacter smithii $(\mathrm{p}=0.0359)$.

\section{Discussion}

The primary cause of obesity is the excess energy intake. An increased energy intake as small as $1 \%$ can induce an increased body weight in the long term (several years) together with possible metabolic alterations [41]. Excessive energy intake is associated with increased nutrient absorption through the intestinal epithelium; and in this process, the intestinal microbiota may play a significant role [42-44]. Changes in intestinal microbiota composition and metabolic capacity may be associated with metabolic changes in the host. For instance, in the laboratory situation of a-xenism, the lack of intestinal microbiota coincides with the accumulation of glycogen produced via gluconeogenesis [45]. Gene expression related to the metabolism of carbohydrate, fat, and amino acids in luminal bacteria are modified in obese individual when comparing intestinal microbial metagenomics of twins with different body weight [46]. It was most believed that the unbalance of Bacteroidetes and Firmicutes has been related to obesity [47], and there are more Firmicutes, less Bacteroidetes, and consequently a 
Feng et al.: Monosodium L-Glutamate and Dietary Fat Differently Modify the

Composition of the Intestinal Microbiota in Growing Pigs

higher Firmicutes/Bacteroidetes ratio in the gastrointestinal tract of obese mice and human $[34,48,49]$. Factors that induce obesity also change Bacteroidetes and Firmicutes in a similar way. High-fat diet modifies intestinal microbiota composition, and increase the ratio of Firmicutes/Bacteroidetes within a small range [50]. In the present study, similar results were found (fig. 4). However, in contrast to previous studies, the ratio of Firmicutes/Bacteroidetes in jejunum was decreased, indicating that the modification of the intestinal microbiota composition may be different depending on the intestinal anatomical segments used compared with the microbiota in feces. No obvious effect of MSG when given alone on the microbiota composition was observed. Another interesting result is that the individual addition of either dietary fat or MSG has no influence on the ratio of Firmicutes/Bacteroidetes in colon, but the ratio of Firmicutes/Bacteroidetes was increased when the two nutrients were added at the same time.

In the present study, several species with energy-harvesting capacity were found to be influenced by dietary fat and MSG. Dietary fat increased the percentage of colonic Methanobrevibacter smithii while MSG had the opposite effect. Methanobrevibacter smithii is a hydrogen-consuming methanogen $[25,47]$, which produces acetic acid and butyric acid by eliminating hydrogen and formic acid to supply energy substrates for colonic epithelial cells. Taking into account that less Methanobrevibacter smithii are present in the gastrointestinal tract of obese individuals [51], the results obtained suggest that there is no simple relationship between the relative amount of this bacteria, dietary fat consumption and obesity. There is however a good correlation between the relative amount of Methanobrevibacter smithii and the relative amount of other bacteria, namely Bacteroides thetaiotaomicron, which have a marked ability to use plant polysaccharides compared with other intestinal microbes $[52,53]$. In the genome of Bacteroides thetaiotaomicron, there are 173 different glycosyl hydrolase activities, which allow Bacteroides thetaiotaomicron to decompose the vast majority of glucosidic bond. Methanobrevibacter smithii can promote Bacteroides thetaiotaomicron to degrade fructose and produce formic acid [54]. The two bacteria can act in synergy for harvesting energy from polysaccharides [55]. Although Bacteroides thetaiotaomicron was not detected using RT-PCR method in the present study, it is possible that dietary fat and MSG may modify the colonization of Methanobrevibacter smithii and thus the amount of polysaccharide utilization in the intestinal tract. This illustrates the difficulty to predict the metabolic and physiological consequences of changes in the composition of the intestinal microbiota.

In the same line of thought, Peptostreptococcus productus has a strong ability to degrade lignin $[56,57]$. Dietary fat increased the percentage of Peptostreptococcus productus among total ileal microbes while fat decreased its relative amount in colon, demonstrating once again that the effect of dietary changes may be different according to the segment of the intestine. Dietary fat may thus enhance the degradation of lignin in the distal part of the small intestine while decreasing the utilization of lignin in the large intestine. MSG decreased the percentage of Peptostreptococcus productus in both jejunum and colon, showing once again complex interactions between these two dietary compounds on the intestinal microbiota.

The species that were most affected by dietary fat and MSG are Faecalibacterium prausnitzii and Fusobacterium prausnitzii. Faecalibacterium prausnitzii has a high capacity to degrade undigested dietary fibers [58-60]. It has been reported that more Faecalibacterium prausnitzii were found in the gut of obese individuals than in their lean counterpart [61], though less studies came to an opposite conclusion [62]. Fusobacterium prausnitzii can efficiently use undigested compounds to produce butyrate used as energy substrate by for colonic epithelial cells [63]. Dietary supplementation with both fat and MSG increases the percentage of these two species by 3-and 6-fold respectively.

Prevotella also have a great ability in fermentation and hydrolysis of dietary fibers to produce acetic acid and propionic acid [64]. Addition of dietary fat increased the percentages of Prevotella in total jejunal, ileal, and cecal microbiota while addition of MSG has no influence 
Feng et al.: Monosodium L-Glutamate and Dietary Fat Differently Modify the

Composition of the Intestinal Microbiota in Growing Pigs

on Prevotella. Clostridium which also play a role in energy harvesting was reported to be present at much different levels in obese individuals than in individuals with low body weight [26]. The fact that dietary fat enhance colonization of Clostridium coccoides group, Clostridium leptum group and Faecalibacterium prausnitzii, while MSG just enhance colonization of Faecalibacterium prausnitzii, strongly suggest that dietary fat and MSG affect the intestinal microbiota of growing pigs via different ways.

The individual body weight has been reported to be closely associated with the diversity of its intestinal microbiota. Indeed, intestinal microbiota diversity is clearly decreased in obese or overweight individuals $[25,26]$. In our study suggests that both dietary fat and MSG can increase the diversity of intestinal microbiota. This result may be considered as somewhat paradoxical given some previously published data. One underlying reason may be related to the different used models. Another reason maybe that dietary fat or MSG enhance the energy and amino acid supply in the gut $[65,66]$, which may promote microbial growth and proliferation.

\section{Conclusion}

The composition of intestinal microbiota and obesity are likely linked by complex relationships. In addition, the metabolic capacity of intestinal microbiota is also an important parameter to be taken into account. In the present study, we have shown that addition of dietary fat and MSG can markedly change the composition of intestinal microbiota, with antagonistic interaction between the two dietary compounds, especially in the colon. Interestingly, dietary fat and MSG promote the colonization of microbes with energy-harvesting capacity in the intestinal tract via different ways, which was consistent with promoted fat deposition in muscle. Dietary fat promoted colonization of Methanobrevibacter smithii, Peptostreptococcus productus, Faecalibacterium prausnitzii, Fusobacterium prausnitzii, bacteria from the Clostridium coccoides and Clostridium leptum groups as well as Roseburia and Prevotella. By contrast, MSG only promoted the colonization of Faecalibacterium prausnitzii and Roseburia. Further work is obviously required in order to establish clear causal links between the dietary changes, the changes in intestinal microbiota composition, the changes in the metabolic capacity of the microbiota and the metabolites released from dietary and endogenous luminal substrates, particularly those which are used by colonocytes and peripheral tissues.

\section{Availability of Supporting Data}

The data sets supporting the results of this article are included within the article and its additional files.

\section{Authors' Contributions}

ZF and YY conceived and designed the experiments. ZF, LW, and DF performed the experiments. ZF analyzed the data. ZF and TL contributed reagents/materials/analysis tools. ZF, FB, and YY wrote the paper.

\section{Acknowledgement}

This work was supported by the Major Program for International Cooperation of the National Natural Science Foundation of China (Grant No.31110103909), the National Natural Science funds (Grant No. 31272463) and key projects of Hunan province natural science funds (Grant No. 12JJ2014). 
Feng et al.: Monosodium L-Glutamate and Dietary Fat Differently Modify the

Composition of the Intestinal Microbiota in Growing Pigs

\section{Disclosure Statement}

The authors declare that they have no competing interests.

\section{References}

1 Xu J, Gordon JI: Honor thy symbionts. Proc Natl Acad Sci U S A 2003;100:10452-10459.

- Hooper LV, Gordon JI: Commensal host-bacterial relationships in the gut. Science 2001;292:1115-1118.

-3 Claus SP, Tsang TM, Wang Y, Cloarec O, Skordi E, Martin FP, Rezzi S, Ross A, Kochhar S, Holmes E, Nicholson JK: Systemic multi-compartmental effects of the gut microbiome on mouse metabolic phenotypes. Mol Syst Biol 2008;4:219.

-4 Björkholm B, Bok CM, Lundin A, Rafter J, Hibberd ML, Pettersson S: Intestinal microbiota regulate xenobiotic metabolism in the liver. PLoS One 2009;4:e6958.

5 Zimmet P, Alberti KG, Shaw J: Global and societal implications of the diabetes epidemic. Nature 2001;414: 782-787.

6 Finucane MM, Stevens GA, Cowan MJ, Danaei G, Lin JK, Paciorek CJ, Singh GM, Gutierrez HR, Lu Y, Bahalim AN, Farzadfar F, Riley LM, Ezzati M, Global Burden of Metabolic Risk Factors of Chronic Diseases Collaborating Group (Body Mass Index): National, regional, and global trends in body-mass index since 1980: system-atic analysis of health examination surveys and epidemiological studies with 960 country-years and 9.1 million participants. Lancet 2011;377:557-567.

7 Speakman JR, O’Rahilly S: Fat: an evolving issue. Dis Model Mech 2012;5:569-573.

8 Popkin BM: Economists' insights into the obesity crisis. Trends Endocrinol Metab 2009;20:417.

9 Barton M: Childhood obesity: a life-long health risk. Acta Pharmacol Sin 2012;33:189-193.

$\rightarrow 10$ Hung HC, Yang YC, Ou HY, Wu JS, Lu FH, Chang CJ: The association between self-reported sleep quality and overweight in a Chinese population. Obesity (Silver Spring) 2013;21:486-492.

11 Tamashiro KL, Hegeman MA, Sakai RR: Chronic social stress in a changing dietary environment. Physiol Behav 2006;89:536-542.

12 Day K, Alfonzo M, Chen Y, Guo Z, Lee KK: Overweight, obesity, and inactivity and urban design in rapidly growing Chinese cities. Health Place 2013;21:29-38.

13 Berridge KC: 'Liking' and 'wanting' food rewards: brain substrates and roles in eating disorders. Physiol Behav 2009;97:537-550.

14 Cui Z, Dibley MJ: Trends in dietary energy, fat, carbohydrate and protein intake in Chinese children and adolescents from 1991 to 2009. Br J Nutr 2012;108:1292-1299.

15 Popkin BM: The shift in stages of the nutrition transition in the developing world differs from past experiences! Public Health Nutr 2002;5:205-214.

16 Beyreuther K, Biesalski HK, Fernstrom JD, Grimm P, Hammes WP, Heinemann U, Kempski O, Stehle P, Steinhart H, Walker R: Consensus meeting: monosodium glutamate-an update. Eur J Clin Nutr 2007;61:304-313.

-17 Narukawa M, Morita K, Uemura M, Kitada R, Oh SH, Hayashi Y: Nerve and behavioral responses of mice to various umami substances. Biosci Biotechnol Biochem 2011;75:2125-2131.

18 He K, Du S, Xun P, Sharma S, Wang H, Zhai F, Popkin B: Consumption of monosodium glutamate in relation to incidence of overweight in Chinese adults: China Health and Nutrition Survey (CHNS). Am J Clin Nutr 2011; 93:1328-1336.

19 Boutry C, Bos C, Matsumoto H, Even P, Azzout-Marniche D, Tomé D, Blachier F: Effects of monosodium glutamate supplementation on glutamine metabolism in adult rats. Front Biosci (Elite Ed) 2011;3:279-290.

$\checkmark 20$ Boutry C, Matsumoto H, Airinei G, Benamouzig R, Tomé D, Blachier F, Bos C: Monosodium glutamate raises antral distension and plasma amino acids after a standard meal in humans. Am J Physiol Gastrointest Liver Physiol 2011;300:G137-G145.

21 Shi Z, Luscombe-Marsh ND, Wittert GA, Yuan B, Dai Y, Pan X, Taylor AW: Monosodium glutamate is not associated with obesity or a greater prevalence of weight gain over 5 years: findings from the Jiangsu Nutrition Study of Chinese adults. Br J Nutr 2010;104:457-463.

-22 Ren X, Ferreira JG, Yeckel CW, Kondoh T, de Aranjo IE: Effects of ad libitum ingestion of monosodium glutamate on weight gain in C57BL6/J mice. Digestion 2011;83:32-36.

23 Cox AJ, West NP, Cripps AW: Obesity, inflammation, and the gut microbiota. Lancet Diabetes Endocrinol 2015; 3:207-215.

-24 Qin J, Li Y, Cai Z, Li S, Zhu J, Zhang F, Liang S, Zhang W, Guan Y, Shen D, Peng Y, Zhang D, Jie Z, Wu W, Qin Y, Xue W, Li J, Han L, Lu D, Wu P, Dai Y, Sun X, Li Z, Tang A, Zhong S, Li X, Chen W, Xu R, Wang M, Feng Q, Gong M, Yu J, Zhang Y, Zhang M, Hansen T, Sanchez G, Raes J, Falony G, Okuda S, Almeida M, Le Chatelier E, Renault P, Pons N, Batto JM, Zhang Z, Chen H, Yang R, Zheng W, Li S, Yang H, Wang J, Ehrlich SD, Nielsen R, Pedersen O, Kristiansen K, Wang J: A metagenome-wide association study of gut microbiota in type 2 diabetes. Nature. 2012; 490:55-60.

25 Eckburg PB, Bik EM, Bernstein CN, Purdom E, Dethlefsen L, Sargent M, Gill SR, Nelson KE, Relman DA: Diversity of the human intestinal microbial flora. Science 2005;308:1635-1638. 
Feng et al.: Monosodium L-Glutamate and Dietary Fat Differently Modify the

Composition of the Intestinal Microbiota in Growing Pigs

26 Nadal I, Santacruz A, Marcos A, Warnberg J, Garagorri JM, Moreno LA, Martin-Matillas M, Campoy C, Martí A, Moleres A, Delgado M, Veiga OL, García-Fuentes M, Redondo CG, Sanz Y: Shifts in Clostridia, Bacteroides and immunoglobulin-coating fecal bacteria associated with weight loss in obese adolescents. Int J Obes (Lond) 2009;33:758-767.

27 Selhub EM, Logan AC, Bested AC: Fermented foods, microbiota, and mental health: ancient practice meets nutritional psychiatry. J Physiol Anthropol 2014;33:2.

28 Tsuei J, Chau T, Mills D, Wan YJ: Bile acid dysregulation, gut dysbiosis, and gastrointestinal cancer. Exp Biol Med (Maywood) 2014;239:1489-1504..

29 Musso G, Gambino R, Cassader M: Interactions between gut microbiota and host metabolism predisposing to obesity and diabetes. Annu Rev Med 2011;62:361-380.

-30 Sartor RB: The intestinal microbiota in inflammatory bowel diseases. Nestle Nutr Inst Workshop Ser 2014;79: 29-39.

-31 Sánchez de Medina F, Romero-Calvo I, Mascaraque C, Martínez-Augustin O: Intestinal inflammation and mucosal barrier function. Inflamm Bowel Dis 2014;20:2394-2404

-32 Musso G, Gambino R, Cassader M: Interactions between gut microbiota and host metabolism predisposing to obesity and diabetes. Annu Rev Med 2011;62:361-380.

33 Russell DW: Cholesterol biosynthesis and metabolism. Cardiovasc Drugs Ther 1992;6:103-110.

-34 Kalliomaki M, Collado MC, Salminen S, Isolauri E: Early differences in fecal microbiota composition in children may predict overweight. Am J Clin Nutr 2008;87:534-538.

-35 Santacruz A, Collado MC, García-Valdés L, Segura MT, Martín-Lagos JA, Anjos T, Martí-Romero M, Lopez RM, Florido J, Campoy C, Sanz Y: Gut microbiota composition is associated with body weight, weight gain and biochemical parameters in pregnant women. Br J Nutr 2010;104:83-92.

-36 Penders J, Thijs C, Vink C, Stelma FF, Snijders B, Kummeling I, van den Brandt PA, Stobberingh EE: Factors influencing the composition of the intestinal microbiota in early infancy. Pediatrics 2006;118:511-521.

-37 Moreira AP, Texeira TF, Ferreira AB, do Carmo Gouveia Peluzio C, de Cássia Gonçalves Alfenas C: Influence of a high-fat diet on gut microbiota, intestinal permeability and metabolic endotoxaemia. Br J Nutr 2012;108: 801-809.

-38 Myles IA, Fontecilla NM, Janelsins BM, Vithayathil PJ, Segre JA, Datta SK: Parental dietary fat intake alters offspring microbiome and immunity. J Immunol 2013;191:3200-3209.

-39 van der Laan JW, Brightwell J, McAnulty P, Ratky J, Stark C; Steering Group of the RETHINK Project: Regulatory acceptability of the minipig in the development of pharmaceuticals, chemicals and other products. J Pharmacol Toxicol Methods 2010;62:184-195.

40 Walter J, Tannock GW, Tilsala-Timisjarvi A, Rodtong S, Loach DM, Munro K, Alatossava T: Detection and identification of gastrointestinal Lactobacillus species by using denaturing gradient gel electrophoresis and species-specific PCR primers. Appl Environ Microbiol 2000;66:297-303.

41 Hill J0: Understanding and addressing the epidemic of obesity: an energy balance perspective. Endocr Rev 2006;27:750-761.

42 Turnbaugh PJ, Ley RE, Mahowald MA, Magrini V, Mardis ER, Gordon JI: An obesity-associated gut microbiome with increased capacity for energy harvest. Nature 2006;444:1027-1031.

-43 Bäckhed F, Ding H, Wang T, Hooper LV, Koh GY, Nagy A, Semenkovich CF, Gordon JI: The gut microbiota as an environmental factor that regulates fat storage. Proc Natl Acad Sci U S A 2004;101:15718-15723.

44 Cani PD, Delzenne NM: Gut microflora as a target for energy and metabolic homeostasis. Curr Opin Clin Nutr Metab Care 2007;10:729-734.

45 Chuang HL, Huang YT, Chiu CC, Liao CD, Hsu FL, Huang CC, Hou CC: Metabolomics characterization of energy metabolism reveals glycogen accumulation in gut microbiota lacking mice. J Nutr Biochem 2012;23:752-758.

-46 Turnbaugh PJ, Hamady M, Yatsunenko T, Cantarel BL, Duncan A, Ley RE, Sogin ML, Jones WJ, Roe BA, Affourtit JP, Egholm M, Henrissat B, Heath AC, Knight R, Gordon JI: A core gut microbiome in obese and lean twins. Nature 2009;457:480-484.

47 Macfarlane GT, Macfarlane S: Human colonic microbiota: ecology, physiology and metabolic potential of intestinal bacteria. Scand J Gastroenterol Suppl 1997;222:3-9.

48 Ley RE, Turnbaugh PJ, Klein S, Gordon JI: Human gut microbes associated with obesity. Nature 2006;444: 1022-1023.

-49 Duncan SH, Lobley GE, Holtrop G, Ince J, Johnstone AM, Louis P, Flint HJ: Human colonic microbiota associated with diet, obesity and weight loss. Int J Obes (Lond) 2008;32:1720-1724.

50 Lam YY, Ha CW, Campbell CR, Mitchell AJ, Dinudom A, Oscarsson J, Cook DI, Hunt NH, Caterson ID, Holmes AJ, Storlien LH: Increased gut permeability and microbiota change associate with mesenteric fat inflammation and metabolic dysfunction in diet-induced obese mice. PLoS One 2012; 7:e34233.

-51 Million M, Maraninchi M, Henry M, Armougom F, Richet H, Carrieri P, Valero R, Raccah D, Vialettes B, Raoult D: Obesity-associated gut microbiota is enriched in Lactobacillus reuteri and depleted in Bifidobacterium animalis and Methanobrevibacter smithii. Int J Obes (Lond) 2012;36:817-825.

-52 Xu J, Bjursell MK, Himrod J, Deng S, Carmichael LK, Chiang HC, Hooper LV, Gordon JI: A genomic view of the human Bacteroides thetaiotaomicron symbiosis. Science 2003;299:2074-2076.

53 Comstock LE, Coyne MJ: Bacteroides thetaiotamicron: a dynamic, niche-adapted human symbiont. Bioessays 2003;25:926-929. 
Feng et al.: Monosodium L-Glutamate and Dietary Fat Differently Modify the Composition of the Intestinal Microbiota in Growing Pigs

-54 Samuel BS, Hansen EE, Manchester JK, Coutinho PM, Henrissat B, Fulton R, Latreille P, Kim K, Wilson RK, Gordon JI: Genomic and metabolic adaptations of Methanobrevibacter smithii to the human gut. Proc Natl Acad Sci U S A 2007;104:10643-10648.

-55 Samuel BS, Gordon JI: A humanized gnotobiotic mouse model of host-archaeal-bacterial mutualism. Proc Natl Acad Sci U S A 2006;103:10011-10016.

-56 Clavel T, Henderson G, Alpert CA, Philippe C, Rigottier-Gois L, Doré J, Blaut M: Intestinal bacterial communities that produce active estrogen-like compounds enterodiol and enterolactone in humans. Appl Environ Microbiol 2005; 71:6077-6085.

57 Clavel T, Henderson G, Engst W, Doré J, Blaut M: Phylogeny of human intestinal bacteria that activate the dietary lignan secoisolariciresinol diglucoside. FEMS Microbiol Ecol 2006;55:471-478.

-58 Wrzosek L, Miquel S, Noordine ML, Bouet S, Chevalier-Curt MJ, Robert V, Philippe C, Bridonneau C, Cherbuy C, Robbe-Masselot C, Langella P, Thomas M: Bacteroides thetaiotaomicron and Faecalibacterium prausnitzii influence the production of mucus glycans and the development of goblet cells in the colonic epithelium of a gnotobiotic model rodent. BMC Biol 2013;11:61.

59 Lopez-Siles M, Khan TM, Duncan SH, Harmsen HJ, Garcia-Gil LJ, Flint HJ: Cultured representatives of two major phylogroups of human colonic Faecalibacterium prausnitzii can utilize pectin, uronic acids, and host-derived substrates for growth. Appl Environ Microbiol 2012;78:420-428.

-60 Benus RF, van der Werf TS, Welling GW, Judd PA, Taylor MA, Harmsen HJ, Whelan K: Association between Faecalibacterium prausnitzii and dietary fibre in colonic fermentation in healthy human subjects. Br J Nutr 2010;104:693-700.

-61 Balamurugan R, George G, Kabeerdoss J, Hepsiba J, Chandragunasekaran AM, Ramakrishna BS: Quantitative differences in intestinal Faecalibacterium prausnitzii in obese Indian children. Br J Nutr 2010;103:335-338.

-62 Furet JP, Kong LC, Tap J, Poitou C, Basdevant A, Bouillot JL, Mariat D, Corthier G, Doré J, Henegar C, Rizkalla S, Clément K: Differential adaptation of human gut microbiota to bariatric surgery-induced weight loss: links with metabolic and low-grade inflammation markers. Diabetes 2010;59:3049-3057.

63 Barcenilla A, Pryde SE, Martin JC, Duncan SH, Stewart CS, Henderson C, Flint HJ: Phylogenetic relationships of butyrate-producing bacteria from the human gut. Appl Environ Microbiol 2000;66:1654-1661.

64 Salyers AA: Bacteroides of the human lower intestinal tract. Annu Rev Microbiol 1984;38:293-313.

65 Nakamura H, Kawamata Y, Kuwahara T, Torii K, Sakai R: Nitrogen in dietary glutamate is utilized exclusively for the synthesis of amino acids in the rat intestine. Am J Physiol Endocrinol Metab 2013;304:E100-E108.

-66 Daniel H, Gholami AM, Berry D, Desmarchelier C, Hahne H, Loh G, Mondot S, Lepage P, Rothballer M, Walker A, Böhm C, Wenning M, Wagner M, Blaut M, Schmitt-Kopplin P, Kuster B, Haller D, Clavel T: High-fat diet alters gut microbiota physiology in mice. ISME J 2014;8:295-308. 